\title{
ARTICLE
}

\section{Evaluation of the radiation dose for whole body in boron neutron capture therapy}

\author{
Kenta Takada $^{\mathrm{a}^{*}}$, Tomonori Isobe ${ }^{\mathrm{a}}$, Hiroaki Kumada ${ }^{\mathrm{a}}$, Tetsuya Yamamoto ${ }^{\mathrm{a}}$, Koichi Shida ${ }^{\mathrm{b}}$, Daisuke Kobayashi ${ }^{\mathrm{b}}$, \\ Yutaro Mori ${ }^{\mathrm{a}}$, Hideyuki Sakurai ${ }^{\mathrm{a}}$ and Takeji Sakae ${ }^{\mathrm{a}}$ \\ ${ }^{a}$ Graduate School of Comprehensive Human Sciences, University of Tsukuba, 1-1-1, Tennodai, Tsukuba-shi, Ibaraki-ken, 305-8575, \\ Japan; ${ }^{b}$ Department of Radiology, University of Tsukuba Hospital,2-1-1, Amakubo, Tsukuba-shi, Ibaraki-ken, 305-8575, Japan
}

\begin{abstract}
Boron neutron capture therapy (BNCT) is a radiation treatment for cancer that uses charged particles from the ${ }^{10} \mathrm{~B}(\mathrm{n}, \alpha)^{7} \mathrm{Li}$ reaction. The dose from the resulting alpha particles and ${ }^{7} \mathrm{Li}$ nuclei is called the boron dose, and is the primary dose delivered to tumor cells. Dose to the tumor and surrounding cells during neutron beam irradiation comes not only from the boron dose, but also from dose resulting from the reaction between neutrons and the elements in living tissue, as well as from leakage photons from the reactor core. Those doses other than the boron dose contribute dose not only to tumor cells, but also to healthy tissue. BNCT is performed in consideration of the results of a comprehensive and accurate dose distribution evaluation, but this evaluation is performed only within the localization of the tumor. In this study, we defined the full-body phantom in Monte Carlo code "PHITS". And we evaluated the dose distribution in full-body phantom resulting from BNCT irradiation. Moreover, we also evaluated the radiation shield material that we proposed for effective techniques to reduce the undesirable radiation exposure dose for non-target area during BNCT.
\end{abstract}

Keywords: BNCT; Monte Carlo; PHITS; whole body; radiation exposure dose; radiation shield

\section{Introduction}

Boron neutron capture therapy $(\mathrm{BNCT})$ is a radiation treatment for cancer that uses charged particles from the ${ }^{10} \mathrm{~B}(\mathrm{n}, \alpha){ }^{7} \mathrm{Li}$ reaction. This treatment involves binding stable isotopes such as ${ }^{10} \mathrm{~B}$ to tumor cells. The isotopes emit charged particles with high linear energy transfer (LET) and short range when irradiated by neutrons, thereby selectively destroying only tumor cells during neutron radiation [1]. Because BNCT can selectively damage cancer cells, it is looked to as a potential therapy for brain tumors that are otherwise difficult to treat. Stable isotopes used in BNCT have high cross sections for neutron capture, so low-energy neutrons can be used. The reaction between ${ }^{10} \mathrm{~B}$ and thermal neutrons yields alpha particles and ${ }^{7} \mathrm{Li}$ nuclei (with in vivo ranges of 10 and $5 \mu \mathrm{m}$, respectively), along with $478 \mathrm{keV}$ prompt gamma rays. This reaction is induced by irradiating the patient with thermal neutrons at energies below $0.4 \mathrm{eV}$, or with epithermal neutrons in the energy range of 0.4 $\mathrm{eV}$ to $10 \mathrm{keV}$ [2]. The dose from the resulting alpha particles and ${ }^{7} \mathrm{Li}$ nuclei is called the boron dose, and in BNCT is the primary dose delivered to tumor cells.

Dose to the tumor and surrounding cells during neutron beam irradiation comes not only from the boron dose, but also from dose resulting from the reaction between neutrons and the elements in living tissue, as well as from leakage photons from the reactor core.
Those doses other than the boron dose contribute not only to tumor cells, but also to healthy tissue. BNCT is performed in consideration of the results of a comprehensive and accurate dose distribution evaluation [3], but this evaluation is performed only within the vicinity of the tumor, and there has been little evaluation of the whole-body effects of the undesirable dose that generally accompanies radiation treatment. This is because full-body CT scans are not performed when developing a treatment planning.

In recent years there has been increased interest in undesirable dose accompanying radiation treatment. There have been reports of cancer risks associated with the secondary neutrons generated in proton therapy [4], as well as of possible effects on pacemakers [5,6], making this an important topic for consideration with regard to future directions for radiation therapy.

In this study, we use the multi-purpose particle and heavy ion transport system (PHITS) [7] and a full-body phantom to examine the BNCT neutron irradiation field. We recreate the full-body dose distribution resulting from BNCT irradiation with the goal of developing effective techniques for reducing radiation exposure during BNCT.

*Corresponding author. Email: k-takada@md.tsukuba.ac.jp 


\section{Materials and methods}

\subsection{The BNCT beam}

In the Monte Carlo code of PHITS, we defined the beam used at the Japan Atomic Energy Agency's Japan Research Reactor No. 4 (JRR-4). We performed criticality calculations for the reactor source information to obtain energy spectra. We then precisely defined the JRR-4 beamline in PHITS, and simulated BNCT treatment. In BNCT treatment, a moderator and cadmium shutter allow the use of a variety of spectra. Irradiation using thermal neutrons is the traditional approach, but in recent years epithermal neutrons have been employed for treating deep tumors. Here, we simulated an epithermal neutron beam (ENB).

\subsection{Full-body phantom}

We created a full-body phantom model for evaluating exposure dose. In this study, all body components were defined as soft tissue as per ICRU 46. To evaluate exposure doses at each body part, we defined dose measurement fields of $1 \mathrm{~cm}$ in diameter throughout the body. Defined body parts were the head (upper, left, right), shoulders, chest, abdomen, pelvis, thighs (left, right), shins (left, right), upper arms (left, right), and wrists (left, right).

\subsection{Evaluation for neutron and photon distribution of full body}

We simulated BNCT for a brain tumor. The phantom was situated with its head near the irradiation aperture (Figure 1), and the PHITS input file was loaded. Neutron and photon distributions were evaluated with the body placed so that the anteroposterior axis was angled $25^{\circ}$ to the left of the beam's central axis. Neutron energy classifications are as per IAEA-TECDOC [8].

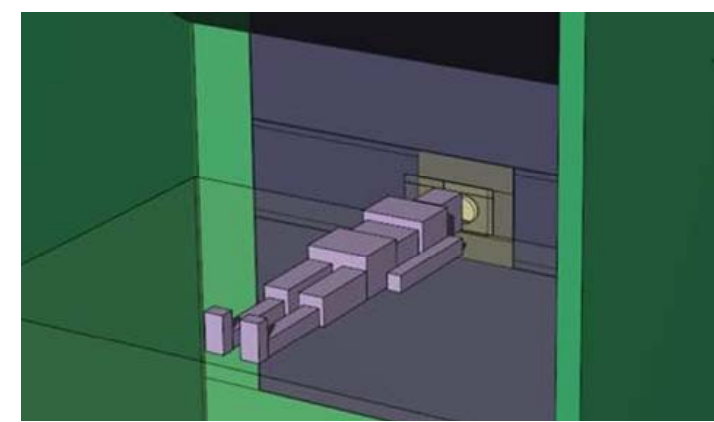

Figure 1. Full-body phantom as defined in PHITS.

"T-track" tally was used to determine the flux of neutrons and photons. Neutron energy bins were distinguished between thermal neutrons $(<0.5 \mathrm{ev})$ and fast neutrons $(>10 \mathrm{keV})$. For photons, the upper limit of energy was set at $10 \mathrm{MeV}$. "T-Heat" tallies were used to determine the heat generation for each region. Calculated heat generation did not consider the relative biological effect (RBE). To consider variation in exposure due to patient orientation, valuation was also performed with the body positioned with the anteroposterior axis $35^{\circ}$ to the left of the beam's central axis.

\subsection{Radiation shielding for dose reduction}

When treating a brain tumor, any radiation to a location other than the brain is considered as an undesirable dose. To test a method for decreasing the dose to areas below the shoulders, we attached radiation shielding to the phantom's shoulders, leaving no gaps. Specific dose reduction materials selected were lead for photons, polyethylene for fast neutrons, and ${ }^{10} \mathrm{~B}$ (in boron trioxide) for thermal neutrons. Figure 2 shows the thickness and proportions of the layers of each of these materials. Boron trioxide and polyethylene in a 1:4 ratio were used for neutron shielding, and the structure was such that emitted photons would be shielded by lead.

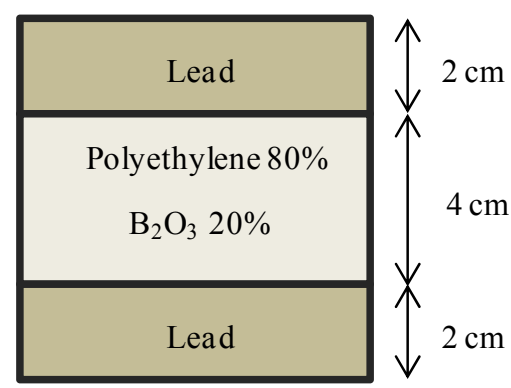

Figure 2. Proposed radiation shielding materials.

\section{Results and discussions}

\subsection{Full-body neutron and photon distributions}

Figure 3 shows the neutron and photon distributions when the full-body phantom is placed by the anteroposterior axis angled $25^{\circ}$ with respect to the beam. Table 1 lists the sums of heat generation for each body part according to T-Heat tally calculations, standardized with heat generation at the top of the head as $100 \%$. Results indicate that undesirable dose by neutrons and photons at the shoulders were confirmed. Little heat was generated in locations farther than the chest. Table 2 lists heat generation for each body part when the body was angled at $35^{\circ}$. This additional $10^{\circ}$ angle increased heat generation at the left side of the head, but resulted in a large decrease of heat generation in the area around the shoulders. Note that we did not consider the boron concentration for any body parts and RBEs for radiations in these calculations. Thus these ratios in table 1,2 were non-precise for dose evaluation as clinical BNCT.

\subsection{Effectiveness of radiation shielding}

The full-body distributions of neutrons and photons indicate a significant undesirable dose at the shoulders. We therefore covered this area with a radiation shield and performed similar calculations to investigate potential reductions in exposure. Figure 4 shows the neutron and photon distributions at around the neck and 


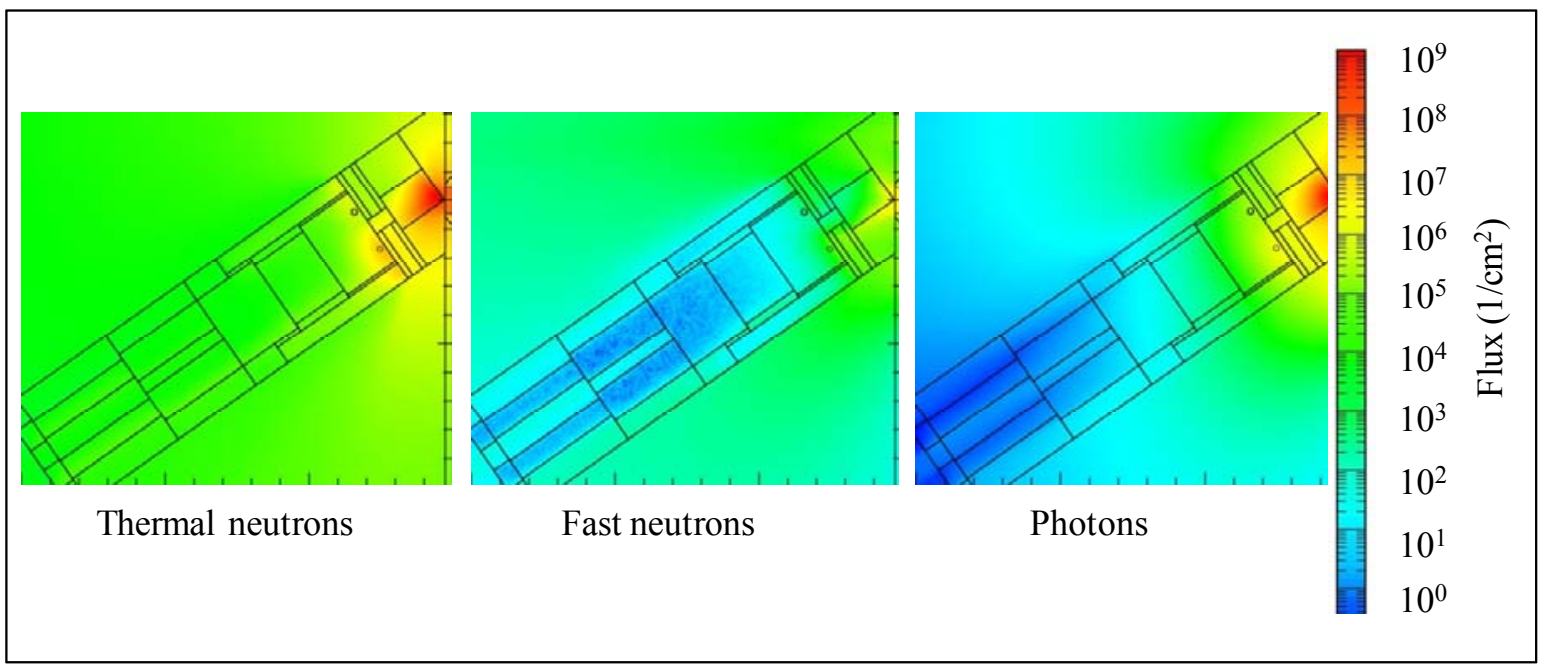

Figure 3. Evaluation of neutron and photon distribution for full-body phantom.

Table 1. Comparison of generated heat at various parts of body. (Anteroposterior axis: $25^{\circ}$ to the left)

\begin{tabular}{lrll}
\hline \multicolumn{4}{c}{ The heat generation of head top was standardized as 100\%. } \\
Head (Left) & $66.8 \%$ & Chest & $4.1 \%$ \\
Head (Right) & $19.8 \%$ & Abdomen & $1.6 \%$ \\
Head (Back) & $25.0 \%$ & Hip & $0.5 \%$ \\
Shoulder (Left) & $37.2 \%$ & Arm (Left) & $2.3 \%$ \\
Shoulder (Right) & $13.0 \%$ & Arm (Right) & $1.5 \%$
\end{tabular}

*The boron concentration was not considered in these ratios.

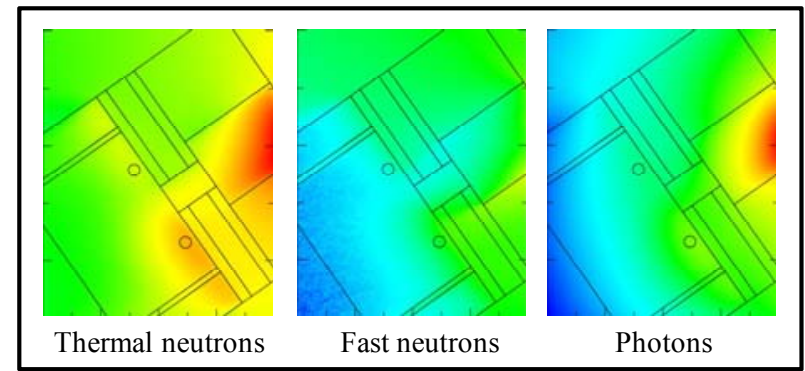

Figure 4. Generated heat distribution around the neck.

(Absent the radiation shield)

the radiation shield is not attached. Figure 5 shows the neutron and photon distribution at same position with radiation shield. Figure 6 shows the comparison of heat generation at the shoulders with the radiation shield both present and absent. The vertical axis shows relative values, where $100 \%$ is the heat generation at the top of the head. As shown in figure 6 , the presence of radiation shielding reduces heat generation to $1 / 12$ of the non-shielded value at the left shoulder, and to $1 / 5$ of the non-shielded value at the right shoulder.

The radiation shielding used in this study consisted of polyethylene to stop fast neutrons, boron to stop thermal neutrons, and lead to stop photons. The thickness of the
Table 2. Comparison of generated heat at various parts of body. (Anteroposterior axis: $35^{\circ}$ to the left)

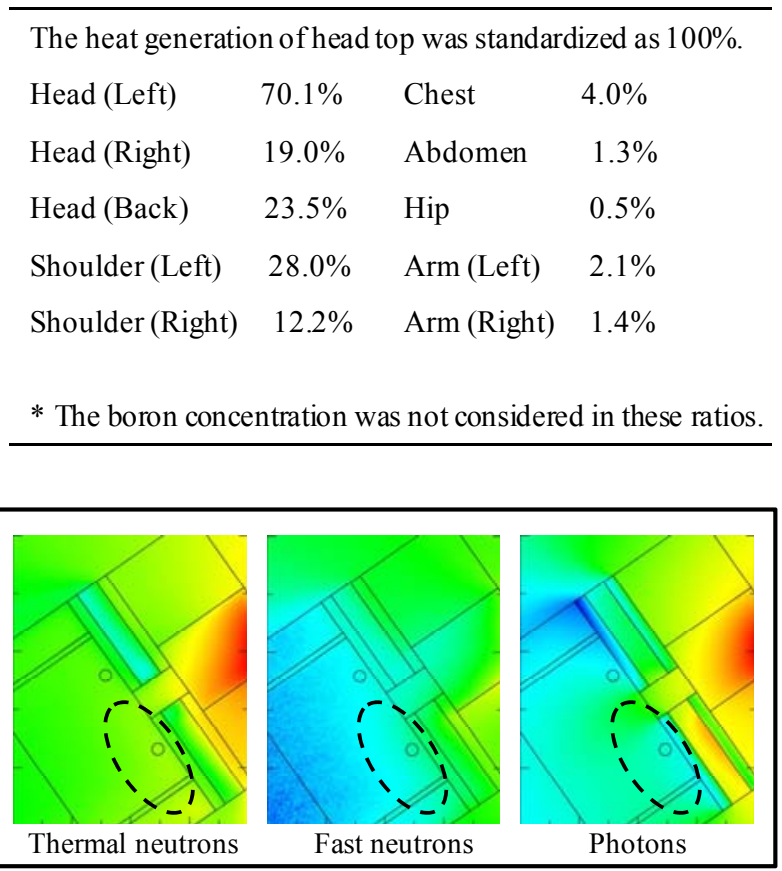

Figure 5. Generated heat distribution around the neck. (Present the radiation shield)

lead to shield for photon was $2 \mathrm{~cm}$. Lead is used in two layers to prevent secondary exposure to prompt gamma rays resulting from the reaction of thermal neutrons and boron. The energy of prompt gamma rays is $478 \mathrm{keV}$. The $1 / 10$ value layer for a $500 \mathrm{keV}$ gamma ray is 1.25 $\mathrm{cm}$, suggesting that the lead is sufficiently thick. In future research we will look for optimal thickness values.

\subsection{Dose evaluation by T-heat tally on PHITS}

In clinical BNCT, "weighted-dose" that considered of compound biological effectiveness factor (CBE factor) 


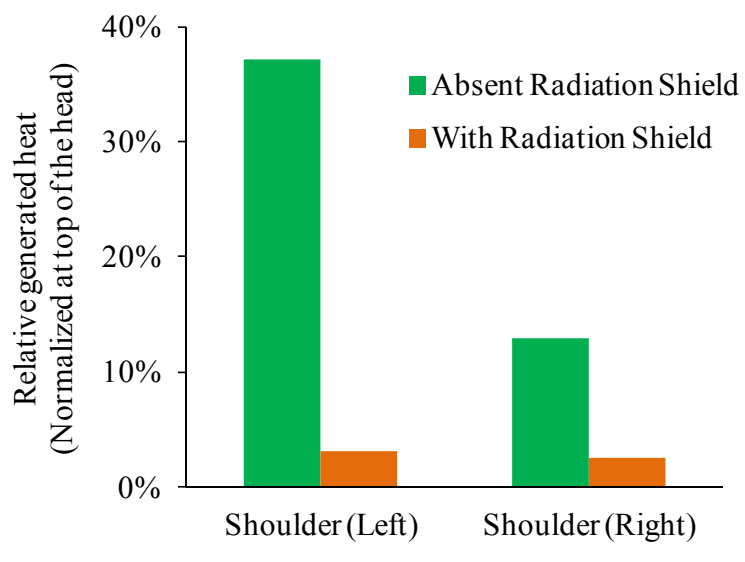

Figure 6. Comparison of heat generation at the shoulders with the radiation shield both present and absent.

and RBEs is used for dose evaluation. Note that we did not consider boron concentration to the tumor or body parts in this study. Moreover T-heat tally can calculate only the physical dose in region of interest. Calculated heat generation was the value that RBE was not considered. Therefore, the ratios of heat generation for each body part to that of head were non-precise for an index of dose evaluation for BNCT. The ratios of heat generation in each parts of the body were overestimated than "weighted-dose". However, the effect to decrease the physical dose of the shoulder by the proposed radiation shield was confirmed.

For the accurate dose evaluation (e.g. based on weighted-dose), the detailed examination that considered boron concentration in the tumor with a computed tomography based full-body model is necessary. This is our limitation for this study.

\section{Conclusion}

We used a simple phantom and PHITS to investigate full-body radiation exposure during BNCT. Results indicated that the utilization of appropriate radiation shielding can reduce undesirable dose in the area around the shoulders.

\section{Acknowledgments}

The authors thank Mr. Koichi Yamanashi, who helped with calculation of PHITS and data analysis.

\section{References}

[1] M.F. Hawthorne and M.W. Lee, A critical assessment of boron neutron capture therapy: an overview, Journal of Neuro-Oncology 62 (2003), pp. 1-5.

[2] P. Ferrari, G. Gualdrini, E. Nava and K.W. Burn, Preliminary evaluations of the undesirable patient dose from a BNCT treatment at the ENEA-TAPIRO reactor, Radiat. Prot. Dosimetry 126 (2007), pp. 636-639.

[3] H. Kumada, K. Yamamoto, T. Yamamoto, K. Nakai, Y. Nakagawa, T. Kageji and A. Matsumura, Improvement of dose calculation accuracy for BNCT dosimetry by the multi-voxelmethod in JCDS, Appl. Radiat. Isot. 61 (2004), pp. 1045-1050.

[4] D.J. Brenner and E.J. Hall, Secondary neutrons in clinical proton radiotherapy: a charged issue, Radiother. Oncol. 86 (2008), pp. 165-170.

[5] J.R. Marbach, M.R. Sontag, J. Van Dyk and A.B. Wolbarst, Management of radiation oncology patients with implanted cardiac pacemakers: report of AAPM Task Group No. 34, American Association of Physicists in Medicine, Med. Phys. 21 (1994), pp. 85-90.

[6] Y. Oshiro, S. Sugahara, M. Noma, M. Sato, Y. Sakakibara, T. Sakae, Y. Hayashi, H. Nakayama, K. Tsuboi, N. Fukumitsu, A. Kanemoto, T. Hashimoto and K. Tokuuye, Proton beam therapy interference with implanted cardiac pacemakers, Int. J. Radiat. Oncol. Biol. Phys. 72 (2008), pp. 723-727.

[7] K. Niita, N. Matsuda, Y. Iwamoto, H. Iwase, T. Sato, H. Nakashima, Y. Sakamoto and L. Sihver, PHITS: Particle and Heavy Ion Transport Code System, Version 2.23, JAEA-Data/Code 2010-022, (2010).

[8] International Atomic Energy Agency, Current Status of Neutron Capture Therapy, IAEA-TECDOC-1223, (2001). 